VoL. 51 (1995) [495-499]

\title{
A NOTE ON GROWTH SEQUENCES OF FINITE SIMPLE GROUPS
}

\author{
Ahmad Erfanian and James Wiegold
}

The aim of this paper is to give a new precise formula for $h(n, A)$, where $A$ is a finite non-abelian simple group, $h(n, A)$ is the maximum number such that $A^{h(n, A)}$ can be generated by $n$ elements, and $n \geqslant 2$. P. Hall gave a formula for $h(n, A)$ in terms of the Möbius function of the subgroup lattice of $A$; the new formula involves a concept called cospread associated with that of spread as explained in Brenner and Wiegold (1975).

\section{INTRODUCTION}

For any finitely generated group $A$, the minimum number of generators of $A$ is denoted by $d(A)$. The growth sequence is the integer sequence $\left\{d\left(A^{n}\right)\right\}$, where $A^{n}$ stands for the $n$th direct power of $A$. The growth sequences of finite groups are known with great accuracy in terms of various parameters $[7,8,9,10]$, and quite a lot is known in the case of finitely generated infinite groups [11].

One of the main theoretical tools in the finite case is a result of $\mathrm{P}$. Hall [5], showing that for a finite non-abelian simple group $A$, and $n \geqslant d(A)$,

$$
d\left(A^{k}\right) \leqslant n \Leftrightarrow k \leqslant h(n, A):=\frac{1}{\mid \text { Aut } A \mid} \sum_{H \leqslant A} \dot{\mu}(H)|H|^{n},
$$

where $\mu$ is the Möbius function of the subgroup lattice of $A$. Of course, Möbius functions can be hard to calculate, even for quite small groups like the alternating group $A_{10}$.

The purpose of this note is to give a somewhat different formula for $h(n, A)$, the new ingredient being the concept of cospread, which grew out of a rich correspondence between Brenner and the second author. (See [1] for results on spread.)

DEFINITION: Let $G$ be any group and $H$ any subgroup of $G$. The cospread of $B$ in $G$ is

$$
c s(H)=\mid\{g: g \in G \quad \text { and } \quad\langle H, g\rangle=G\} \mid .
$$

Received 4 August 1994

Copyright Clearance Centre, Inc. Serial-fee code: 0004-9729/95 \$A2.00+0.00. 
Clearly, $c s(H)$ is sometimes zero, but we believe it to be non-zero for every nontrivial subgroup of a finite simple group $A$. (Equivalently, $A$ has spread one in the parlance of [1].)

The main result is essentially a systematisation of methods used in previous articles, more particularly [5] and [6].

Theorem 1. Let $A$ be a finite non-abelian simple group. Then, for every $n \geqslant 0$,

$$
h(n+1, A)=\sum_{\left(x_{1}, \ldots, x_{n}\right) \in \Delta_{n}} \frac{c s\left(\left\langle x_{1}, \ldots, x_{n}\right\rangle\right)}{\left|C_{\text {Aut } A}\left(x_{1}, \ldots, x_{n}\right)\right|},
$$

where, for each $i, \Delta_{i}$ is any set of representatives of the Aut $A$-classes of ordered $i$-vectors of elements of $A$.

One can imagine many variants of this result, for example the following. (We shall omit the proof.)

THEOREM 2. For any finite simple group $A$ and integers $m, n$ with $1 \leqslant m<n$,

$$
h(n, A)=\sum_{\left(x_{1}, x_{2}, \ldots, x_{m}\right) \in \Delta_{m}} \frac{c s_{m, n-m}\left(\left\langle x_{1}, \ldots, x_{m}\right\rangle\right)}{\left|C_{\text {Aut } A}\left(x_{1}, x_{2}, \ldots, x_{m}\right)\right|}
$$

where, for any subgroup $H$,

$$
c s_{m, n-m}(H)=\mid\left\{\left(x_{1}, \ldots, x_{n-m}\right): x_{i} \in A \quad \text { and } \quad\left\langle H, x_{1}, \ldots, x_{n-m}\right\rangle=A\right\} \mid .
$$

Some applications of Theorem 1 will be given in Section 3.

\section{Proof of TheOREM 1}

Firstly, we recall [6] that the ordered $m$-vectors

$$
\begin{gathered}
\left(a_{11}, a_{12}, \ldots, a_{1 m}\right) \\
\vdots \\
\left(a_{n 1}, a_{n 2}, \ldots, a_{n m}\right)
\end{gathered}
$$

of elements of $A$ generate $A^{m}$ if and only if $A=\left\langle a_{1 i}, \ldots, a_{n i}\right\rangle$ for each $i$ and the $m$ column vectors $\left(a_{1 i}, \ldots, a_{n i}\right)^{\prime}$ are inequivalent under the action of Aut $A$.

Let $t$ denote the number on the right-hand side of (1). We shall display $t$ Aut $A$ inequivalent generating $(n+1)$-vectors for $A$, and show that every generating $(n+1)$ vector is equivalent to one of them. This will be enough for our purposes. Suppose that $\Delta_{n}$ consists of $n$-vectors

$$
\left[\begin{array}{c}
x_{11} \\
\vdots \\
x_{1 n}
\end{array}\right], \ldots,\left[\begin{array}{c}
x_{r 1} \\
\vdots \\
x_{r n}
\end{array}\right]
$$


so that $\left|\Delta_{n}\right|=r$. Clearly, every generating $(n+1)$-vector of $A$ is Aut $A$-equivalent to one of the form

$$
\left[\begin{array}{c}
x_{i 1} \\
\vdots \\
x_{i n} \\
g
\end{array}\right]
$$

for suitable $g \in A$ and suitable $i$; let us denote that vector by $v(i, g)$ for short. Two vectors $v(i, g)$ and $v(j, h)$ are Aut $A$-equivalent if and only if $i=j$ and there is an $\alpha$ in Aut $A$ with $x_{i o}^{\alpha}=x_{i}$ for $s=1,2, \ldots, n$ and $g^{\alpha}=h$. Also, for each $i$, the number of $g$ such that $v(i, g)$ is a generating $(n+1)$-vector is $c s\left(\left\langle x_{i 1}, \ldots, x_{i n}\right\rangle\right)$; finally, for fixed $i$ the number of Aut $A$-inequivalent $v(i, g)$ is

$$
\frac{c s\left(\left\langle x_{i 1}, \ldots, x_{i n}\right\rangle\right)}{\left|C_{\mathrm{Aut} A}\left(x_{i 1}, \ldots, x_{i n}\right)\right|}
$$

Thus, there are in all $t_{1}+\ldots+t_{r}=t$ inequivalent generating $(n+1)$-vectors of the form $v(i, g)$, and every generating $(n+1)$-vector is equivalent to one of them. This completes the proof of Theorem 1.

\section{Applications}

From Theorem 1 we get, for non-abelian simple group $A$,

$$
h(2, A)=\sum_{l=1}^{r} \frac{c s\left(x_{i}\right)}{\left|C_{\mathrm{Aut} A}\left(x_{i}\right)\right|},
$$

where $\left\{x_{1}, \ldots, x_{r}\right\}$ is a complete set of representatives of the Aut $A$-classes of elements of $A$. (In fact this number is $1 /(|A u t A|) \sum_{x \in A} c s(x)$, of course; however, the formula obtained here makes calculations easier). For example, for $A_{5}$ we have four Aut $A_{5}$ classes, represented by $1, x_{2}=(1,2,3,4,5), x_{3}=(1,2,3), x_{4}=(1,2)(3,4)$; and it is easy to check by hand that $c s\left(x_{1}\right)=0$

$$
\begin{aligned}
& c s\left(x_{2}\right)=50, \quad\left|C_{\mathrm{Aut} A_{5}}\left(x_{2}\right)\right|=5, \\
& c s\left(x_{3}\right)=36, \quad\left|C_{\mathrm{Aut} A_{5}}\left(x_{3}\right)\right|=6 \text {, } \\
& \operatorname{cs}\left(x_{4}\right)=24, \quad\left|C_{\text {Aut } A_{5}}\left(x_{4}\right)\right|=8
\end{aligned}
$$

so that we recover the famous value $[5]$ for $h\left(2, A_{5}\right)$ :

$$
h\left(2, A_{5}\right)=50 / 5+36 / 6+24 / 8=19 .
$$


Hall's method depends on knowing the Möbius functions of the subgroup lattices, a rare phenomenom. Using a computer to check cospreads, we have made the following new evaluations (among others):

$$
\begin{aligned}
h\left(2, A_{7}\right) & =916, & h\left(2, A_{8}\right)=7,448, \\
h\left(2, A_{9}\right) & =77,015, & h\left(2, A_{10}\right)=793,827, \\
h\left(2, M_{11}\right) & =6,478 . &
\end{aligned}
$$

Finally, it is probable that cospread calculations can be used in conjunction with the classification theorem to show that $h(2, A) \rightarrow \infty$ as $|A| \rightarrow \infty$ and $A$ runs over finite non-abelian simple groups. Indeed, all that needs confirmation is that every such group has spread one; that is, for every $a \in A \backslash 1$ there exists $b \in A$ such that $A=\langle a, b\rangle$. This is known for projective special linear groups [1], alternating groups $[1,2]$, the Mathieu groups $M_{11}$ and $M_{12}$ [3] and the Suzuki groups [4].

We begin with a simple result.

LemMa. Let $A$ be a finite 2-generator group, say $\langle a, b\rangle$. Then cs $(a) \geqslant\left|C_{\mathrm{Aut} A}(a)\right|$.

Proof: For $\alpha \in C_{\text {Aut } A}(a)$ we have $A=\langle a, b\rangle^{\alpha}=\left\langle a, b^{\alpha}\right\rangle$. On the other hand the $b^{\alpha}$ with $\alpha \in C_{\text {Aut } A}(a)$ are all different; if $b^{\alpha}=b^{\beta}$ for $\alpha, \beta \in C_{\text {Aut } A}(a)$, then $\alpha \beta^{-1}$ fixes $a$ and $b$ and thus is the identity of $A$.

Of course, one expects $c s(a)$ to be much larger than $\left|C_{\mathrm{Aut} A}(a)\right|$ in general. However, the lemma is sufficient to enable us to estabish our final result.

Theorem 5. Let $\left\{A_{\alpha}\right\}_{\alpha \in J}$ be an infinite set of finite non-abelian simple groups of spread one. Then $h\left(2, A_{\alpha}\right) \rightarrow \infty$ as $\left|A_{\alpha}\right| \rightarrow \infty$.

PROOF: This result is a very elementary consequence of the Lemma, Theorem 1 and the fact that the exponent of $A_{\alpha}$ tends to infinity with $\left|A_{\alpha}\right|$, the last being a consequence of the classification theorem. All we have to note is that $h(2, A) \geqslant r$ for a non-abelian simple group $A$ of spread one ( $r$ being the number of Aut $A$-classes of elements), and that elements of different orders are Aut $A$-inequivalent.

\section{References}

[1] J.L. Brenner and J. Wiegold, 'Two generator groups I', Michigan Math. J. 22 (1975), 53-64.

[2] J.L. Brenner and J. Wiegold, 'Two generator groups II', Bull. Austral. Math. Soc. 22 (1980), 113-124.

[3] J.L. Brenner, R.M. Guralnick and James Wiegold, 'Two generator groups III', Contemp. Math. 33 (1984), 82-89.

[4] M.J. Evans, 'A note on two-generator groups', Rocky Mountain J. Math. 17 (1987), 887-889. 
[5] P. Hall, 'The Eulerian functions of a group', Quart. J. Math. Oxford 7 (1936), 134-151.

[6] D. Meier and J. Wiegold, 'Growth sequences of finite groups V', J. Austral. Math. Soc. Ser. A 31 (1981), 374-375.

[T] J. Wiegold, 'Growth sequences of finite groups', J. Austral. Math. Soc. 17 (1974), 133-141.

[8] J. Wiegold, 'Growth sequences of finite groups II', J. Austral. Math. Soc. 20 (1975), 225-229.

[9] J. Wiegold, 'Growth sequences of finite groups III', J. Austral. Math. Soc. 25 (1978), 142-144.

[10] J. Wiegold, 'Growth sequences of finite groups IV', J. Austral. Math. Soc. 29 (1980), 14-16.

[11] James Wiegold and J.S. Wilson, 'Growth sequences of finitely generated groups', Arch. Math. 30 (1978), 337-343.

School of Mathematics

University of Wales

College of Cardiff

Senghennydd Road

Cardiff CF2 4 YH

Wales

United Kingdom 